\title{
The New Sesquizygotic Twins and More: Exotic Twin Types/Twin Research Reviews: Parental Affection and Co-Twins' Personality; Prenatal Demise in Twin Pregnancies; Heteropaternal Superfecundation; Selective Feticide in Dichorionic Twins/In the News: Identical Twin Oscar Winner; 'Superfecundated' Twins with Gay Fathers; Partly Living Apart; Which Twin Committed the Crime?
}

\author{
Nancy L. Segal \\ Department of Psychology, California State University, Fullerton, CA, USA
}

\begin{abstract}
The second recorded case of sesquizygotic (SQZ) twins is summarized. It is suspected that SQZ twins result from the fertilization of an egg by two separate spermatozoa, followed by division of the fertilized ovum. Next, recent studies examining how differences in parental affection affect co-twins' personality profiles, the prenatal demise of fetuses during twin pregnancy, a forensic case of heteropaternal superfecundation and selective feticide in dichorionic twins are reviewed. Finally, summaries of newsworthy items featuring an identical twin Oscar winner, a case of 'superfecundated' twins born to a gay couple, identical twins who try partly living apart and new molecular techniques to distinguish an innocent identical twin from his or her guilty co-twin are presented.
\end{abstract}

\section{The New Sesquizygotic Twins and More: Exotic Twin Types}

Virtually all books, articles, essays and commentaries on twinning refer to two types of twins: monozygotic (MZ or identical) and dizygotic (DZ or fraternal). It is further explained that $\mathrm{MZ}$ twins result when a single fertilized egg divides between the 1st and 14th postconceptional day. This event results in MZ twins who share $100 \%$ of the genes, barring errors in cell replication, and who are always of the same sex. In contrast, DZ twins result when two simultaneously released eggs are fertilized at the same time by two separate spermatozoa. DZ twins share half of their genes on average, by descent, just like ordinary siblings born years apart. DZ twins may be of the same sex or opposite sex.

Over the years, another twin type, namely 'polar body twins,' has been recognized (Segal, 2017). It is often labeled the 'third twin type' by parents and twins in need of a way to describe their children or themselves as falling somewhere between $\mathrm{MZ}$ and DZ pairs. Such twins usually look a lot alike, but are not exactly alike in either appearance or behavior. The label 'third twin type' is, however, invoked much too quickly, and in the absence of DNA studies of family members, it is impossible to demonstrate whether it accurately classifies a given twin pair. At the same time, there is a

Author for correspondence: Nancy L. Segal, Email: nsegal@fullerton.edu Cite this article: Segal N. (2019) The New Sesquizygotic Twins and More: Exotic Twin Types/Twin Research Reviews: Parental Affection and Co-Twins' Personality; Prenatal Demise in Twin Pregnancies; Heteropaternal Superfecundation; Selective Feticide in Dichorionic Twins/In the News: Identical Twin Oscar Winner; 'Superfecundated' Twins with Gay Fathers; Partly Living Apart; Which Twin Committed the Crime? Twin Research and Human Genetics 22: 195-198 https://doi.org/10.1017/thg.2019.16 sound scientific basis for such twins, best described in the classic work of Bulmer (1970). In fact, Bulmer outlined a series of twin types (some theoretical) that would range in genetic relatedness. Based on his work, I explain in Segal (2017, pp. 289-291) that:

Were one to rank $M Z, D Z$ and polar twins in increasing order of average genetic similarity, the order would be primary oocytary (least similar), $D Z$, secondary oocytary, uniovular dispermatic and $M Z$ (most similar). The primary oocytary polar body twins would be less alike than typical DZ twins, so the 'third twin type' does not always fall between $M Z$ and twin pairs when it comes to similarity ... some of these twin types are theoretically possible, but have not been identified in the scientific literature.

In summary, many people assume that there is just one kind of polar body twinning (i.e., the fertilization of a polar body and mature ovum by separate spermatozoa), but Bulmer shows that the situation is more complex.

As the biological underpinnings of twinning have become better understood, there is the realization that there are more than just two types of twins, but these pairs can be classified within the broader categories of MZ or DZ. Some exotic types of twins are gaining attention and could well be more frequent than presumed. In the event that such pairs do occur more commonly, they could impact the results from extant and future MZ-DZ twin comparisons.

The impetus for this article was the announcement of the birth of the second known set of sesquizygotic (SQZ) twins. The report was published by an Australian team (Gebbett et al., 2019) and has been covered extensively in the media (BBC, 2019). SQZ twins, labeled popularly as 'semi-identical' twins by the BBC, are formed when two separate spermatozoa penetrate a single ovum that 
divides. The recently identified pair was carried by a 28 -year-old first-time mother. A monochorionic-diamniotic twin pregnancy was detected at 6 weeks of gestation, indicative of MZ twins; however, the unusual nature of her pregnancy was revealed at 14 weeks: she was carrying one male fetus and one female fetus. Noninvasive DNA testing showed that each twin's maternally derived genomes were identical, whereas the twins were chimeric for their paternally derived genomes, for which they showed $78 \%$ overlap.

The biological process by which SQZ twinning is thought to occur is called heterogonesis, that is, 'chimerism arising at the juncture of zygotic division' following dispermic fertilization of the ovum (Gebbett et al., 2019, p. 842). A diagram accompanying the scientific article depicts the different steps in this chain of events.

The frequency of SQZ twins is unknown, but its rarity seems likely by the failure of the Australian team to detect sesquizygosity among an additional 968 DZ twin pairs whom they purposely screened. The only previous report involving a twin pair comprised one hermaphrodite and one normal male of unknown chorionicity. The most recent case included a normal male and female. Unfortunately, the female co-twin suffered from a right brachial artery thromboembolism just after delivery, requiring amputation of her arm at 4 weeks of age. Her abnormality was thought to have occurred just before birth, so appeared unrelated to her unusual biological origin.

Some selected studies and case reports of unusual twinning are presented below; see, also, the case of heteropaternal twinning described in the Twin Research Reviews section of this article that follows. A recent paper illustrating the different types of twins by McNamara et al. (2016) is also informative.

I first heard suggestive evidence of polar body twinning described by Fisher and Polesky (1979) at a professional conference. Sixteen sets of twins conceived via the fertility drug clomid were fully concordant for red blood cell antigens, but discordant for other highly heritable traits (e.g., hair and eye color). Significantly increased rates of physical defects such as clubfoot and urinary tract abnormalities were observed among the twins in this sample. The clomid-linked mechanism(s) that might produce these unusual twins, as well as some evolutionary considerations, are discussed in a later paper (Fischer, 1990).

In a case report, an evidence of polar body twinning was provided by the detection of two maternally derived chromosome sets and fertilization by two separate spermatozoa (Bieber et al., 1981). Specifically, the twins resulted from the fertilization of a normal haploid ovum and diploid first-meiotic division polar body. The twins, which developed in a shared chorion, consisted of a normal male twin (XY) and a triploid acardiac co-twin (XXX). The presence of the XXX karyotype indicated that the twins originated from different conceptional events and different spermatozoa.

A more recent case report documented a presumed rare case of superfecundation, following transfer of two embryos, resulting in quintuplets (Peigné et al., 2011). The couple in question had experienced 5 years of infertility, so the woman underwent several trials of in vitro fertilization (IVF), with two embryos eventually transferred successfully. A transvaginal ultrasound examination at 4 weeks into the pregnancy revealed five intrauterine gestational sacs. The couple indicated that they had had intercourse the day after oocyte retrieval, an event that appeared linked to the higher-order multiple conception. A decision was made to selectively reduce the quintuplets to twins at 12 weeks' gestation. The investigators were uncertain as to how their patient was able to ovulate following oocyte retrieval, noting that only one other such case has been reported in the literature.
The presumed rarity of the various extraordinary twin types described above should not make researchers complacent about their representation in a modest-sized study. For example, a relatively high proportion of SQZ pairs or superfecundated twin participants could affect the findings by amplifying the expected similarity of the DZ twin sample, thereby reducing heritability estimates. For now, it would be prudent for researchers to be aware of such twins and to report these cases when they are detected.

\section{Twin Research Reviews}

\section{Parental Affection and Twins' Personality}

Sources of influence on co-twins' personality differences have been of interest to researchers in many behavioral science fields. Dunkel et al. (2018) applied a life history (LH) approach in a study of how parental affection may be linked to $\mathrm{MZ}$ co-twin differences across the Big Five personality traits of openness, conscientiousness, extraversion, agreeableness and neuroticism. LH theory, grounded in an evolutionary theoretical framework, makes predictions about trade-offs in how individuals allocate resources and energy; parental investment is seen as one influence on a child's LH strategy. Individual differences in each person's LH approach are also believed to encompass individual differences in personality. In this study, greater affection from mothers and fathers toward a particular twin was predicted to be associated with a relatively slow $\mathrm{LH}$ strategy, as would be evidenced by that twin's higher scores in plasticity, stability and a general personality factor.

Data were provided by $349 \mathrm{MZ}$ twin pairs, identified from among the 7108 participants in the Midlife Development in the United States (MIDUS I) study. The mean age of the full sample $(N=7108)$ was 46 years $(S D=13.2)$ and ranged between 24 and 75 years. Zygosity was assigned by responses to questions regarding physical similarities and confusion by others, as well as birth information from a physician and/or a report of DNA testing. Personality traits were assessed by self-ratings of adjectives.

Maternal and paternal affection were positively associated with conscientiousness, extraversion, agreeableness, plasticity, stability and the general personality factor, although only paternal affection was linked to openness. Consistent with LH predictions, co-twins reporting greater affection had a higher general personality factor, supporting a slower LH strategy. It was noted that differences in paternal affection were more strongly associated with general personality factor differences than differences in maternal affection; this finding was explained by the suggestion that paternal affection is a significant LH cue. Finally, it was recognized that many environmental influences comprise the range of nonshared effects on personality. As the MZ twins age, these other factors, such as peer group expectations, may assume more significant roles.

\section{Prenatal Demise in Twin Pregnancies}

Monochorionicity places two-thirds of MZ twins at increased risk for fetal death. According to researchers at the University of Wisconsin, the most common causes of death among this subset of MZ twin pairs include twin-to-twin transfusion syndrome, acardia and twin-twin disruption (Korlesky \& McPherson, 2019). Using the 3137 records from the Wisconsin Stillbirth Program, gathered between 1983 and 2017, 175 relevant twin pregnancies were identified. Key findings were that monochorionic (MC) twins had the lowest co-twin survival rate $(30 / 95,32 \%)$, whereas DZ twins had the highest co-twin survival rate $(17 / 24,71 \%)$, and twins whose zygosity was undetermined (UZ) were intermediate $(27 / 56$, 
48\%). MZ twin losses occurred at an earlier mean gestational age (27 weeks) than those of DZ twins and UZ twins ( 30 weeks and 28 weeks, respectively).

Other findings are of interest. The fetal weights of miscarried and stillborn twins were smaller than predicted at given gestational ages, especially among the UZ twin pairs. Interestingly, placental weights were smaller for DZ twins; in fact, over half were below the 10th percentile. It was suspected that while the MC placentas were larger, they appeared to be inefficient, leading to growth retardation and other abnormalities. The use of ultrasound for placental evaluation, including blood flow, was suggested.

\section{Heteropaternal Superfecundation}

A single case of heteropaternal superfecundation from among 78 cases of disputed paternity involving twins was reported by Chinese investigators (Sun et al., 2016). This case came to professional attention when the alleged father of both twins requested testing, due to his uncertainty as to the twins' paternity. (The specific reasons underlying his concern were not disclosed.) The twins in question, delivered at 36 weeks' gestation, were both male. Their birth weight and birth length were $2750 \mathrm{~g}$ and $47.2 \mathrm{~cm}$ and $2550 \mathrm{~g}$ and $46.2 \mathrm{~cm}$, respectively. DNA was extracted from buccal swabs provided by both parents and both twins.

Genetic analysis revealed that the client could have fathered only one twin, based on their concordance for 15 autosomal markers and $17 \mathrm{Y}$ chromosome markers. In contrast, nine genetic inconsistencies were found among the autosomal markers and 12 genetic inconsistencies were found among the $\mathrm{Y}$ chromosome markers in the case of the other twin. The biological relatedness of the mother to both twins was confirmed. The researchers believe (as do I) that the frequency of this type of twinning is probably more common than is believed, given (1) advances in reproductive technologies, and (2) the increase in multiple partners and coital frequency in some populations; for example, the recent practice of 'temporary couples' occurs in segments of the Chinese population. Such temporary couples include migrant workers who seek short-term sexual partners due to extended separation from their spouses (Women of China, 2014).

\section{Selective Feticide in Dichorionic Twins}

Selective feticide (SF) is sometimes performed in twin pregnancies in the event that one twin shows a genetic or congenital anomaly, or a woman's previous pregnancy included complications linked to premature delivery. It was noted that co-twins are discordant for anatomical or chromosomal abnormalities in nearly $85 \%$ of multiple pregnancies.

An analysis of data on feticide from 44 cases involving dichorionic (DC) twins was undertaken by Kim et al. (2019) in South Korea. Two study groups were fairly evenly divided between two indications for feticide given above (Group I: Anomaly, and Group II: Birth History). The cases were gathered between December 2006 and January 2007 in the Department of Obstetrics and Gynecology at the CHA University in South Korea. The median age at which SF was performed was 14 weeks for Group I and 12 weeks for Group II. The rate of fetal loss, median gestational age at delivery and birth weight did not differ between groups. Only one pregnancy loss from the procedure occurred in the case of Group I. In addition, when the cases were organized according to the timing of SF (before 15 weeks; at 15 weeks or later), differences in the fetal loss rate, median gestational age at delivery and preterm delivery rate were not observed.
However, the mean birth weight of babies delivered after 15 weeks was significantly decreased, relative to those delivered earlier. This counterintuitive result was explained by the $22 \%$ increase in the preterm delivery rate in the second group.

SF was first described in 1978 , but is not widely practiced. The investigators suggested that SF may be a reasonable alternative for some twin pregnancies for improving the chance of delivering at least one healthy child if there is considerable risk to the pregnancy, in general.

\section{In the News}

\section{Identical Twin Oscar Winner}

Rami Malek, the Oscar winner for his starring role in the 2018 film Bohemian Rhapsody, has an identical twin brother, Sami (Perrie, 2019). Sami works in the field of education in the Los Angeles area and loves the work that he does. These identical twins, now aged 37 , claim to look a lot less alike than they did when they were younger. Many people feel that these twins lead very separate lives, given that one is a movie star and the other is an instructor. However, both professions involve speaking before crowds.

\section{'Superfecundated' Twins with Gay Fathers}

Superfecundation occurs when a woman releases two eggs simultaneously that are then fertilized at different times within the fourday window of opportunity (see research reviews above). The sperm may be contributed by the same male or by different males. In the event that different males are involved, the genetic relatedness between the twins is $25 \%$, on average, making them genetically equivalent to half-siblings. Twins recognized as such are referred to as superfecundated twins. The frequency of such twins is presumed to be rare, but in reality their frequency is unknown because only pairs identified as such, variously due to differences in size, appearance or paternity uncertainty, come to light (Segal, 2017).

A gay couple from Great Britain, Simon and Graeme BerneyEdwards, recently made headlines across the globe when news of their unique children was released (Fraser, 2019). The two fathers donated sperm to a 32-year-old Canadian woman who agreed to carry the babies for them. Their hope was that each man would be the biological father of one twin, and while that outcome could not be guaranteed, it happened - half of the eggs were fertilized with the sperm from Simon and half were fertilized by the sperm from Graeme. The procedure took place in the USA because it is not allowed in the UK. The male-female twins, Calder and Alexandra, were born healthy after 36 weeks' gestation.

\section{Partly Living Apart}

Identical female twins, Dale and Hannah LaPlace, were raised on a horse farm in upstate New York. Their next stop together was Seton Hall University in South Orange, New Jersey where they shared a two-bedroom apartment. Their subsequent transition took them to separate apartments, but they came together again in a two-bedroom apartment in a New Jersey building with a view of Manhattan. According to the twins, during that time they shared most of their meals and clothing - not surprising, given that identical twins have similar preferences for food and attire (Cohen, 2018).

When the LaPlace twins turned 31, they decided to move again, due to the building's maintenance problems. Their initial plan was to share a two-bedroom apartment until Hannah challenged that idea, opting instead for two one-bedroom apartments. She 
explained that she did not wish to eventually be known as part of a middle-aged identical twin pair whose members wear only matching outfits. (Note that the photograph of the LaPlace twins that accompanied the article about them shows two young women in the same lavender slacks and similar white blouses.) Perhaps they feel societal pressures to differentiate when, in fact, they are really more comfortable being themselves. Despite their now separate apartments, they share meals, clothes, keys and a pet cat.

\section{Which Twin Committed the Crime?}

For many years, genetic technology did not allow forensic experts to clearly distinguish an innocent identical twin from his or her identical co-twin (Zimmer, 2019). Cases involving sexual offense, theft and paternity languished in the courts. (Ironically, when paternity cannot be assigned in a twin case, it is still possible to identify a child's paternal grandmother!) A test developed by German researchers in the mid-2000s provided information on the thousands of short tandem repeat markers (STRs) in each twin's genome, but was still unable to tell identical twins apart. Then, in 2014, researchers changed direction, focusing instead on postnatal mutations that might differ between the twins. The new test proved successful in an analysis of adult identical male twins, one of whom had a particular mutation that his co-twin did not have, but which he transmitted to his child (WeberLehman et al., 2014).

I have described this research elsewhere, but I believe it is in the news again because of a 2018 decision that found one of two twins guilty in a rape case that had arisen in 1999. The judge believed that the science behind the new genetic test was sound, but argued for replication and the need to educate involved jurors. She also called for increased scientific detail to be presented in a peer-reviewed journal for additional study; in fact, a paper did appear in 2014 (see Weber-Lehman et al., 2014) and another was published more recently (Krawczak et al., 2018). Commenting on the test, Harvard University geneticist Steven A. Carroll urged additional analysis of mutational differences between twins using large twin samples from different centers (see Zimmer, 2019).

It has been estimated that twins are responsible for $1 \%$ of crimes. That is a relatively small number, but given that many cases remain unsolved, it is likely that a significant proportion might now be settled.

\section{References}

BBC. (2019). Semi-identical twins 'identified for only the second time.' $B B C$ Health. Retrieved from https://www.bbc.com/news/health-47371431

Bieber, F. R., Nance, W. E., Morton, C. C., Brown, J. A., Redwine, F. O., Jordan, R. L., \& Mohanakumar, T. (1981). Genetic studies of an acardiac monster: Evidence of polar body twinning in man. Science, 213, 775-777.

Bulmer, M. G. (1970). The biology of twinning in man. Oxford: Clarendon Press.
Cohen, J. (2018). Twins decide to go their separate ways, eight stories apart. New York Times, p. 12.

Dunkel, C. S., Nedelec, J. L., \& van der Linden, D. (2018). Using monozygotic twin differences to examine the relationship between parental affection and personality: A life history account. Evolution and Human Behavior, $39,52-58$.

Fischer, K. (1990). A rapid evolution mechanism may contribute to changes in sex ratio, multiple birth incidence, frequency of auto-immune disease and frequency of birth defects in clomid conceptions. Medical Hypotheses, 31, $59-65$.

Fischer, K. M., \& Polesky, H. F. (1979). Polar body conceptions and clomid twins. In Paper presented at the Annual Meeting of the American Society of Human Genetics, Minneapolis, MN.

Fraser, C. (2019). Seeing double. The Sun. Retrieved from https://www.thesun. co.uk/news/8287975/british-twins-different-dads-fertility-treatment/.

Gebbett, M. T., Laporte, J., Sekar, R., Nandini, A., McGrath, P., Sapkota, Y., .. Fisk, N. M. (2019). Molecular support for heterogonesis resulting in sequizygotic twinning. The New England Journal of Medicine, 380, 842-849.

Kim, M. S., Na, E. D., Kang, S., Shin, S. Y., Lim, B. B., Kim, H., \& Moon, M. J. (2019). Transabdominal selective feticide in dichorionic twins: Ten years' experience at a single center. Journal of Obstetrics and Gynaecology Research, 45, (2), 299-305.

Korlesky, C., \& McPherson, E. (2019). Early demise of twins in a cohort of stillbirths and second trimester miscarriages. American Journal of Medical Genetics, 179A, 350-355.

Krawczak, M., Budowle, B., Weber-Lehmann, J., \& Rolf, B. (2018). Distinguishing genetically between the germlines of male monozygotic twins. PLoS Genetics, 14, e1007756.

McNamara, H. C., Kane, S. C., Craig, J. M., Short, R. V., \& Umstad, M. P. (2016). A review of the mechanisms and evidence for typical and atypical twinning. American Journal of Obstetrics and Gynecology, 214, 172-191.

Peigné, M., Andrieux, J., Deruelle, P., Vuillaume, I., \& Leroy, M. (2011). Quintuplets after a transfer of two embryos following in vitro fertilization: A proved superfecundation. Fertility and Sterility, 95, 2124.e13-6.

Perrie, S. (2019). Rami Malek has an identical twin brother who leads a very different life. LAD Bible. Retrieved from http://www.ladbible.com/entertainment/ celebrity-rami-malek-has-identical-twin-brother-who-leads-a-very-differentlife-20190118.

Segal, N. L. (2017). Twin mythconceptions: False beliefs, fables, and facts about twins. San Diego, CA: Elsevier.

Sun, M., Zhang, X. N., Wu, D., Lu, Q. B., \& Wu, Y. M. (2016). A case study of heteropaternal superfecundation in a pair of Chinese twins. Australian Journal of Forensic Sciences, 50, 341-344.

Weber-Lehman, J., Schilling, E., Gradl, G., Richter, D. C., Wiehler, J., \& Rolf, B. (2014). Finding the needle in the haystack: differentiating 'identical' twins in paternity testing and forensics by ultra-deep next generation sequencing. Forensic Science International, 9, 42-46.

Women of China. (2014). 'Temporary couples' challenge Chinese traditional marriage morally. Retrieved from http://www.womenofchina.cn/ womenofchina/html1/special/gender_events/16/8631-1.htm. (Original source was the Contemporary Gold Newspaper; translation was provided by womenofchina.cn.

Zimmer, C. (2019). A new DNA test can identify the right twin. New York Times, p. D6. 